\title{
SOME RESULTS ON CRUMPLED CUBES $\left({ }^{1}\right)$
}

\author{
BY \\ LLOYD L. LININGER
}

Introduction. This paper deals with some problems on crumpled cubes. In [6] R. H. Bing raised the following questions: (1) Is the union of a crumpled cube and a 3-cell topologically $S^{3}$ ? (2) Under what conditions will the union of two crumpled cubes sewn together along their boundaries be $S^{3}$ ? (3) Under what conditions will the union of two copies of the same crumpled cube sewn together along their boundaries by the identity homeomorphism be $S^{3}$ ? In [2] Bing proves that the union of two copies of the Solid Alexander Horned Sphere sewn together along their boundaries by the identity homeomorphism is $S^{3}$. In [1] B. J. Ball proves that there exists a crumpled cube $C$ such that two copies of $C$ sewn together along their boundaries (not by the identity homeomorphism) is not topologically $S^{3}$. In [7] Casler proves that the union of two copies of the Solid Alexander Horned Sphere sewn together along their boundaries is $S^{3}$.

In this paper it is proved in $\$ 2$ that question (1) has an affirmative answer. This result has recently been obtained independently by Norman Hosay [9]. Using this result, it is shown in $\S 3$ that if $C$ is a crumpled cube and $B$ is a unit ball in $E^{3}$, then there exists an upper semi-continuous decomposition $G$ of $B$ such that (1) $B / G$ is homeomorphic to $C$, and (2) if $g$ is a nondegenerate element of $G$, then $g$ is point-like and the intersection of $g$ with the boundary of $B$ is a onepoint set. Corollary 2 of $\S 3$ shows that if $C$ and $D$ are crumpled cubes, $S$ is a tame 2-sphere in $S^{3}$, and $C$ and $D$ are sewn together along their boundaries, then there exists an upper semi-continuous decomposition $G$ of $S^{3}$ such that the intersection of each nondegenerate element of $G$ with $S$ is a one-point set and $S^{3} / G$ is homeomorphic to the union of $C$ and $D$.

In $\S 4$ it is shown that if two copies of a particular "bad" crumpled cube are sewn together by the identity homeomorphism along their boundaries the result is $S^{3}$, and it is shown that if two copies of a particular "nice" crumpled cube are sewn together by the identity homeomorphism along their boundaries the result is not $S^{3}$.

1. Notation and terminology. If $A$ is a set in a topological space, then $\mathrm{Cl} A$ and Bd $A$ denote the closure and boundary, respectively, of $A$. If $S$ is a 2-sphere in

Presented to the Society, January 26, 1964; received by the editors February 3, 1964.

(1) This paperconsists of the author's doctoral dissertation at the State University of Iowa, prepared under the supervision of Professor S. Armentrout. The author wishes to express his appreciation to Professor Armentrout for his assistance and encouragement. 
$E^{3}$, then Int $S$ and Ext $S$ denote the interior and exterior, respectively, of $S$. The usual metric for $E^{3}$ is $d$.

A set $C$ is a crumpled cube if and only if $C$ is homeomorphic to the union of a 2-sphere and its interior in $E^{3}$.

A space $X$ is a disk-with-holes if and only if $X$ is a disk or there exist disjoint disks $D_{1}, \cdots, D_{n}$ in the interior of a disk $D$ such that $X$ is homeomorphic with $D-\bigcup_{i=1}^{n} \operatorname{Int} D_{i}$.

For terms not defined in this paper the reader may see [4] or [6].

2. The sum of a crumpled cube and a 3-cell. In this section it is proved that the sum of a crumpled cube and a 3-cell is topologically $S^{3}$. Theorem 1 is used to obtain this result. The proof of Theorem 1 given here relies heavily on the Side Approximation Theorem proved by Bing. For completeness this theorem is stated below.

Bing's Side APPROXIMATION TheOREM. For each 2-sphere $S$ in $E^{3}$, each positive number $e$, and each component $U$ of $E^{3}-S$, there esists a homeomorphism $h$ from $S$ onto a polyhedral 2-sphere such that (1) if $x$ is in $S$, then $d(x, h(x))<e$, (2) there exists a finite collection of disjoint disks $D_{1}, \cdots$, and $D_{n}$ on $h[S]$, each of diameter less than e such that $h[S]-\bigcup_{i=1}^{n} D_{i} \subset U$, and (3) there exists a finite collection of disjoint disks $E_{1}, \cdots$, and $E_{m}$ on $S$, each of diameter less than e such that $S-\bigcup_{i=1}^{m} E_{i}$ is contained in Int $h[S]$ if $U$ is the exterior of $S$ or is contained in $\operatorname{Ext} h[S]$ if $U$ is the interior of $S$ [4].

THEOREM 1. If $C$ is a crumpled cube in $E^{3}$ with boundary $S, A$ is a compact subset of Int $C$, and $e$ is a positive number, then there exist a homeomorphism $h^{\prime}$ from $C$ into $E^{3}$ and a polyhedral 2-sphere $S^{\prime}$ such that $S^{\prime}$ is homeomorphically within e of $S, h^{\prime}[C] \subset \operatorname{Int} S^{\prime}$, if $x \in C$ then $d\left(x, h^{\prime}(x)\right)<e$, and if $x \in A$ then $h^{\prime}(x)=x$.

Proof. There exists a positive number $e^{\prime}$ such that if $T$ is a 2-sphere within $e^{\prime}$ of $S$, then $A \subset \operatorname{Int} T$. Let $e^{\prime \prime}$ denote $(1 / 11) \min \left\{e, e^{\prime},(1 / 10)\right.$ diam $\left.C\right\}$. Since $S$ is a 2-sphere, then by Bing's Side Approximation Theorem, there exists a polyhedral 2-sphere $S_{1}$ such that (a) $S_{1}$ is homeomorphically within $e^{\prime \prime}$ of $S$, (b) there exists a finite number of disjoint disks $E_{1}, \cdots$, and $E_{m}$ on $S_{1}$, each of diameter less than $e^{\prime \prime}$, such that $\mathrm{Cl}\left(S_{1}-\bigcup_{i=1}^{m} E_{i}\right)$ is contained in $\operatorname{Ext} S$, and (c) there exists a finite number of disjoint disks $D_{1}, \cdots$, and $D_{n}$ on $S$, each of diameter less than $e^{\prime \prime}$, such that $\mathrm{Cl}\left(S-\bigcup_{i=1}^{n} D_{i}\right)$ is contained in Int $S_{1}$. For each $i, i=1,2, \cdots, m$, let $K_{i}$ denote a polyhedral disk such that (a) Bd $K_{i}=\mathrm{Bd} E_{i}$, (b) $K_{i}-\operatorname{Bd} K_{i}$ is contained in Ext $S_{1}$, (c) $\left(S_{1}-\bigcup_{i=1}^{m} E_{i}\right) \cup\left(\bigcup_{i=1}^{m} K_{i}\right)$ is a 2 -sphere $S^{\prime}$, and (d) $S^{\prime}$ is homeomorphically within $e^{\prime \prime}$ of $S$.

It will be shown that there exist a polyhedral 3-manifold-with-boundary $M$ and a homeomorphism $h$ with the following properties: (1) $(S \cup \operatorname{Int} S) \subset \operatorname{Int} M$, (2) for each $i, i=1,2, \cdots, m$, no simple closed curve in $M$ links $\mathrm{Bd} E_{i}$, (3) if $D_{i}^{\prime}$ is a polyhedral disk such that $\mathrm{Bd} E_{i}=\mathrm{Bd} D_{i}^{\prime}$ then there does not exist a 2-sphere in $M$ that contains $D_{i}^{\prime}$ in its interior, (4) $M-S^{\prime}$ has one and only one component 
$V$ with diameter more than one half the diameter of $C$, (5) $S^{\prime}, S_{1}$, and $M$ are in relative general position, (6) if $W$ is a component of $M \cap S^{\prime}$ such that $W$ does not separate $M$, and $W_{1}, W_{2}, \cdots$, and $W_{k}$ are components of $M \cap S^{\prime}$ such that (i) $\bigcup_{i=1}^{k} W_{i}$ does not separate $M$, (ii) $\bigcup_{i=1}^{k} W_{i} \cup W$ does separate $M$, and (iii) $W \cup \bigcup_{i=1}^{k} W_{i}$ is contained in some component of $\mathrm{Cl}(M-V)$, then there exists a simple closed curve $J$ in $M$ such that $J$ intersects $\bigcup_{i=1}^{k} W_{i}$ in a one-point set, $J$ intersects $W$ in a one-point set, and the diameter of each component of $J \cap V$ is less than $e^{\prime \prime}$, (7) if $x$ is in $V \cap \operatorname{Int} S_{1}$, then $h(x)=x$, (8) $h$ is from $M$ into Int $S^{\prime}$, and (9) for each $x$ in $M, d(x, h(x))$ is less than $11 e^{\prime \prime}$. This will complete the proof of Theorem 1 since if $h^{\prime}$ denotes the restriction of $h$ to $C$ then $h^{\prime}$ and $S^{\prime}$ satisfy the conclusion of Theorem 1 .

There exists a positive number $e_{1}$ such that if $T$ is a triangulation of $E^{3}$ with mesh $e_{1}, N$ is the union of all 3-simplexes of $T$ which intersect $C$, and $M$ is the union of all 3-simplexes is the 2nd barycentric subdivision of $T$ which intersect $N$, then $M$ will be a polyhedral 3-manifold-with-boundary which satisfies properties (1)-(6).

The proof that there exists a homeomorphism $h$ from $M$ into Int $S^{\prime}$ that satisfies conditions (7), (8) and (9) is broken into three parts. Let $F_{1}, \cdots$, and $F_{p}$ be the components of $M-V$. In Part I it is proved that if for some $j$, there exists a positive integer $i$ less than or equal to $p$ such that $(\mathrm{Cl} V) \cap F_{i}$ is contained in $K_{j}$, then there exists a homeomorphism $h_{i}$ from $M$ into $E^{3}$ such that $h_{i}\left[F_{i}\right] \subset \operatorname{Int} S^{\prime}$, $h_{i}[M]$ satisfies conditions (1)-(6), if $V^{\prime}$ is the large component of $h_{i}[M]-S^{\prime}$, then $h_{i}[M]-V^{\prime}$ has at most $p-1$ components, and if $x$ is in $V \cap \operatorname{Int} S_{1}$ then $h_{i}(x)=x$. In Part II the following is proved: If for each $j$ such that $j \leqq p$, there does not exist an integer $i$ such that $F_{i} \cap \mathrm{Cl} V$ is contained in $K_{j}$, then there exists an integer $k, 1 \leqq k \leqq m$, and a homeomorphism $h_{1}$ from $M$ into $E^{3}$ such that $h_{1}[M]$ satisfies conditions (1)-(7), if $V^{\prime \prime}$ denotes the large component of $h_{1}[M]-S^{\prime}$, then $h_{1}[M]-V^{\prime \prime}$ has at most $p$ components, and $h_{1}\left[F_{1}\right] \cap \mathrm{Cl} V^{\prime \prime}$ is contained in $K_{k}$. In Part III, by using induction and Parts I and II it is shown that there exists a homeomorphism $h$ with the desired properties.

PROOF OF PART I. In this part, it is supposed that for some $j$, there exists an integer $i$ such that $(\mathrm{Cl} V) \cap F_{i}$ is contained in $K_{j}$. A homeomorphism $h_{i}$ from $M$ into $E^{3}$ will be constructed such that $h_{i}\left[F_{i}\right] \subset \operatorname{Int} S_{2}^{\prime}, h_{i}[M]$ satisfies conditions (1)-(7), and if $V^{\prime}$ denotes the large component of $h_{i}[M]-S^{\prime}$ then $h_{i}[M]-V^{\prime}$ has at most $p-1$ components.

Let $X_{1}^{1}, \cdots, X_{t(1)}^{1}$ be the distinct components of $F_{i}-K_{j}$ such that for each $u$, $\left(\mathrm{Cl} X_{u}^{1}\right) \cap \mathrm{Cl} V \neq \varnothing$, and if $W$ is a component of $F_{i}-K_{j}$ such that $(\mathrm{Cl} W) \cap \mathrm{Cl} V \neq \varnothing$, then there exists a $z$ such that $W=X_{z}^{1}$. Let $X_{1}^{2}, \cdots$, and $X_{t(2)}^{2}$ be the distinct components of $F_{i}-\mathrm{Cl}\left(K_{j} \cup\left[\bigcup_{k=1}^{t(1)} X_{k}^{1}\right]\right)$ such that for each $u$ less than or equal to $t(2),\left(\mathrm{Cl} X_{u}^{2}\right) \cap \mathrm{Cl}\left(V \cup \bigcup_{k=1}^{t(1)} X_{k}^{1}\right) \neq \varnothing$. Since $F_{i}-K_{j}$ has only a finite number of components, there exists an integer $r$ such that if the above process is continued, for each $u$ and $w, X_{u}^{w} \neq \varnothing$ and $F_{i}=\operatorname{Cl}\left(\bigcup_{w=1}^{r} \bigcup_{u=1}^{t(w)} X_{u}^{w}\right)$. 
For each $u$ and $w$ such that $w$ is greater than $1,\left(\mathrm{Cl} X_{u}^{w}\right) \cap \mathrm{Cl}\left(\bigcup_{k-1}^{t(w-1)} X_{k}^{w-1}\right.$ is a collection of disk-with-holes. Let $Y_{w u l}, \cdots$, and $Y_{w u q(w, u)}$ denote the components of $\left(\mathrm{Cl} X_{u}^{w}\right) \cap\left(\mathrm{Cl}\left[\bigcup_{j=1}^{t(w-1)} X_{j}^{w-1}\right]\right)$ and let $Y_{w u}$ denote $\bigcup_{j=1}^{q(w, u)} Y_{w u j}$. For each $w, u$ and $z$, let $T_{w u z}$ be a disk in $K_{j}^{\prime}$ such that (1) $Y_{w u z} \subset T_{w u z}$, and (2) Bd $T_{w u z} \subset \mathrm{Bd} Y_{w u z}$. Let $T_{w u z}^{\prime}$ be a slight thickening of $T_{w u z}$ with the following properties: (1) $T_{w u z}^{\prime}$ is polyhedral and topologically equivalent to $T_{w u z} \times[0,1]$, (2) $T_{w u z}^{\prime}$ is disjoint from the 0-skeleton of $M$, and (3) If $T_{w u v} \subset T_{w u z}$ then $T_{w u v} \subset \operatorname{Int} T_{w u z}$. Let $T_{w u}^{\prime}$ denote $\bigcup_{z=1}^{q(w, u)} T_{w u z}^{\prime}$, let $T_{w u z}^{\prime \prime}$ denote the component of $T_{w u z}^{\prime}-K_{j}$ that intersects $\bigcup_{u=1}^{t(w-1)} X_{u}^{w-1}$, let $T_{w u}^{\prime \prime}$ denote $\bigcup_{z=1}^{q(w, u)} T_{w u z}^{\prime \prime}$, and let $T_{w}^{\prime \prime}$ denote $\bigcup_{u=1}^{t(w)} T_{w u}^{\prime \prime}$. The remainder of the proof of Part $I$ is now broken into two steps.

Step I. In Step I the following is proved: There exists a homeomorphism $f_{1}$ from $M$ into $E^{3}$ with the following properties: (1) $f_{1}[M]$ has properties (1)-(7), (2) If $V^{\prime}$ is the large component of $f_{1}[M]-S^{\prime}$, then $f_{1}[M]-V^{\prime}$ has at most $p$ components, (3) $f_{1}\left[F_{1}\right]$ is contained in $\left(\operatorname{Int} S^{\prime}\right) \cup\left(F_{1}-\mathrm{Cl} X_{1}^{w}\right) \cup T_{w 1}^{\prime \prime}$, and (4) If $k \neq 1$, then either $\left(f_{1}\left[\mathrm{Cl} X_{k}^{w}\right]\right) \cap K_{j}=f_{1}\left[\left(\mathrm{Cl} X_{k}^{w}\right) \cap K_{j}\right]$ or $\left(f_{1}\left[\mathrm{Cl} X_{k}^{w}\right]\right) \cap K_{j}=\varnothing$.

Proof of Step I. Case 1. $Y_{w 1}=Y_{w 11}$. Let $Y_{w 1}^{*}$ denote the disk on the boundary of $T_{w 1}^{\prime \prime}$ that is disjoint from $K_{j}$ and corresponds to $Y_{w 1}$. See Figure 1. A homeo-

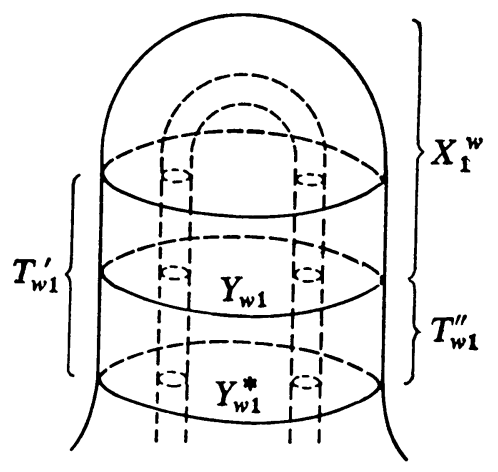

FIGURE 1

morphism $f_{1}$ is constructed as follows: Let $W_{1}, \cdots$, and $W_{g(1)}$ be the components of $M-\mathrm{Bd} T_{w 1}^{\prime \prime}$, and assume Int $S_{1} \cap V$ is contained in $W_{1}$ and $X_{1}^{w}=W_{2}$. If $x$ is in $W_{1}$ then $f_{1}(x)=x$. If $\left(\mathrm{Cl} W_{1}\right) \cup \operatorname{Int} T_{w 1}^{\prime \prime}=\varnothing$ and $W_{1}$ is contained in the unbounded component of $E^{3}-\left(T_{w 1}^{\prime \prime} \cup\left(\mathrm{Cl} X_{1}^{w}\right)\right)$, then $f_{1}(x)=x$. If $W=\left\{W_{i}: W_{i} \cap \operatorname{Int} T_{w 1}^{\prime \prime}=\varnothing\right.$ and either $W_{i}=W_{2}$ or $W_{i}$ is not contained in the unbounded component of $\left.E^{3}-\left(T_{w 1}^{\prime \prime} \cup\left(\mathrm{Cl} X_{1}^{w}\right)\right)\right\}$ and $W^{\prime}=\bigcup\left\{\mathrm{Cl} W_{i}\right.$ : there exists an integer $s$ such that $W_{s} \in W$ and $\left.\mathrm{Cl} W_{s} \cup \mathrm{Cl} W_{i} \neq \varnothing\right\}$, then $f_{1}\left[W^{\prime}\right]$ is contained in $T_{w 1}^{\prime \prime}$ and $f_{1}$ leaves $W^{\prime}$ pointwise fixed on $\mathrm{Bd} T_{w 1}^{\prime \prime}$. Each of the remaining $W_{k}$ 's (if there are any) is contained in $T_{w 1}^{\prime \prime}$ and the homeomorphism $f_{1}$ can be extended to them as shown in Figure 2.

Case 2. $Y_{w 1}=Y_{w 11} \cup \cdots \cup Y_{w 1 q(w, 1)}$. There exists a simple closed curve $J$ on Bd $M$ such that (1) $J \subset \mathrm{Cl}\left(V \cup \bigcup_{k=1}^{w-1} \bigcup_{u=1}^{t(k)} X_{u}^{k}\right)$, (2) The diameter of each compo- 


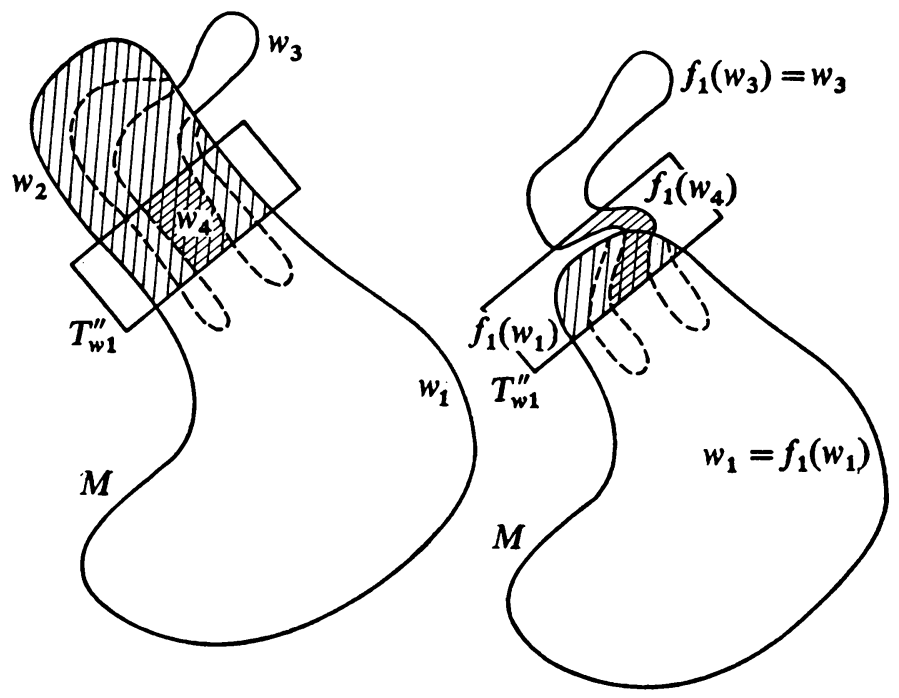

FIGURE 2

nent of $J \cup V$ is less than $e$, and (3) $J \cap Y_{w 11}$ is a one-point set, and it can be assumed that $J \cap Y_{w 12}$ is a one-point set while $J$ is disjoint from each of $Y_{w 13}, \cdots$, and $Y_{w 1 q(w, 1)}$. Shrink $F_{1}$ slightly so that $J \cap F_{1}$ misses the modified $F_{1}$ and if $J$ intersects $V$, pull $J$ off $V$ slightly. See Figure 3. Then there exists a homeomor-
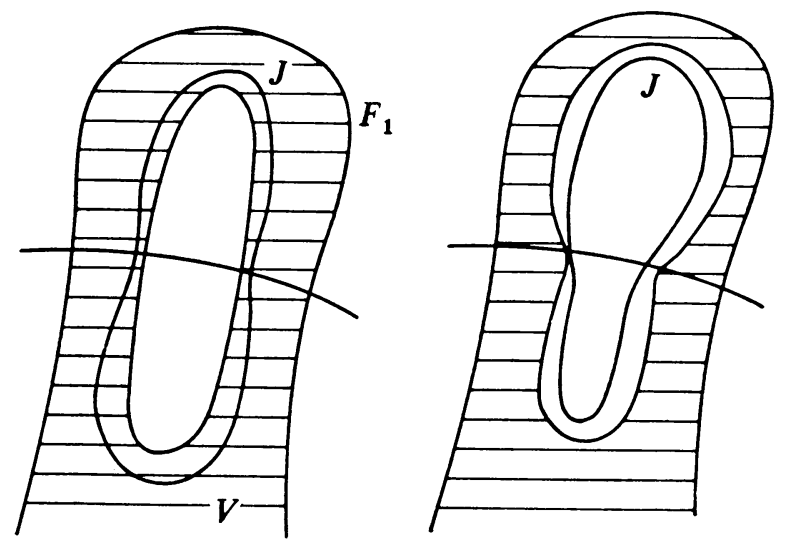

FIGURE 3

phism $f^{*}$ from the modified $M$ into $E^{3}$ with the following properties: (1) If $x$ is in $M-T_{w 12}$ then $f^{*}(x)=x$, (2) $f^{*}$ maps $T_{w 12} \cap$ [modified $M$ ] as shown in Figure 4. Note that $J \cap\left(E^{3}-T_{w 12}\right)$ is the center of $f^{*}\left[T_{w 12} \cap\right.$ modified $\left.M\right]$ $\cap\left(E^{3}-T_{w 12}\right)$, and (3) $f^{*}[$ modified $M]$ satisfies conditions (1)-(7), and if $V^{*}$ is the large component of $f^{*}[\operatorname{modified} M]-S^{\prime}$, then $f^{*}[\operatorname{modified} M]-V^{*}$ has at most $p$ components. After $q(w, 1)-1$ repetitions of this argument, the modified $Y_{w 1}$ is contained in $Y_{w 11}$. See Figure 5. An argument like the one given 

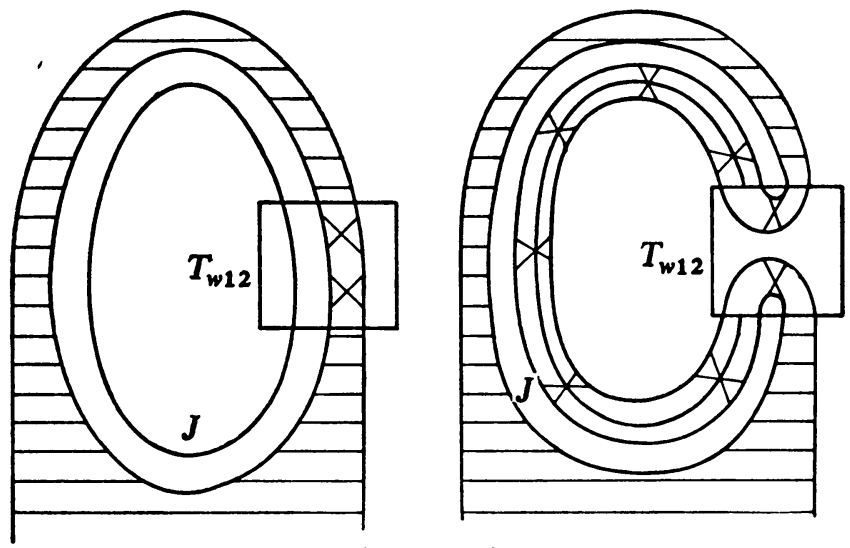

FIGURE 4
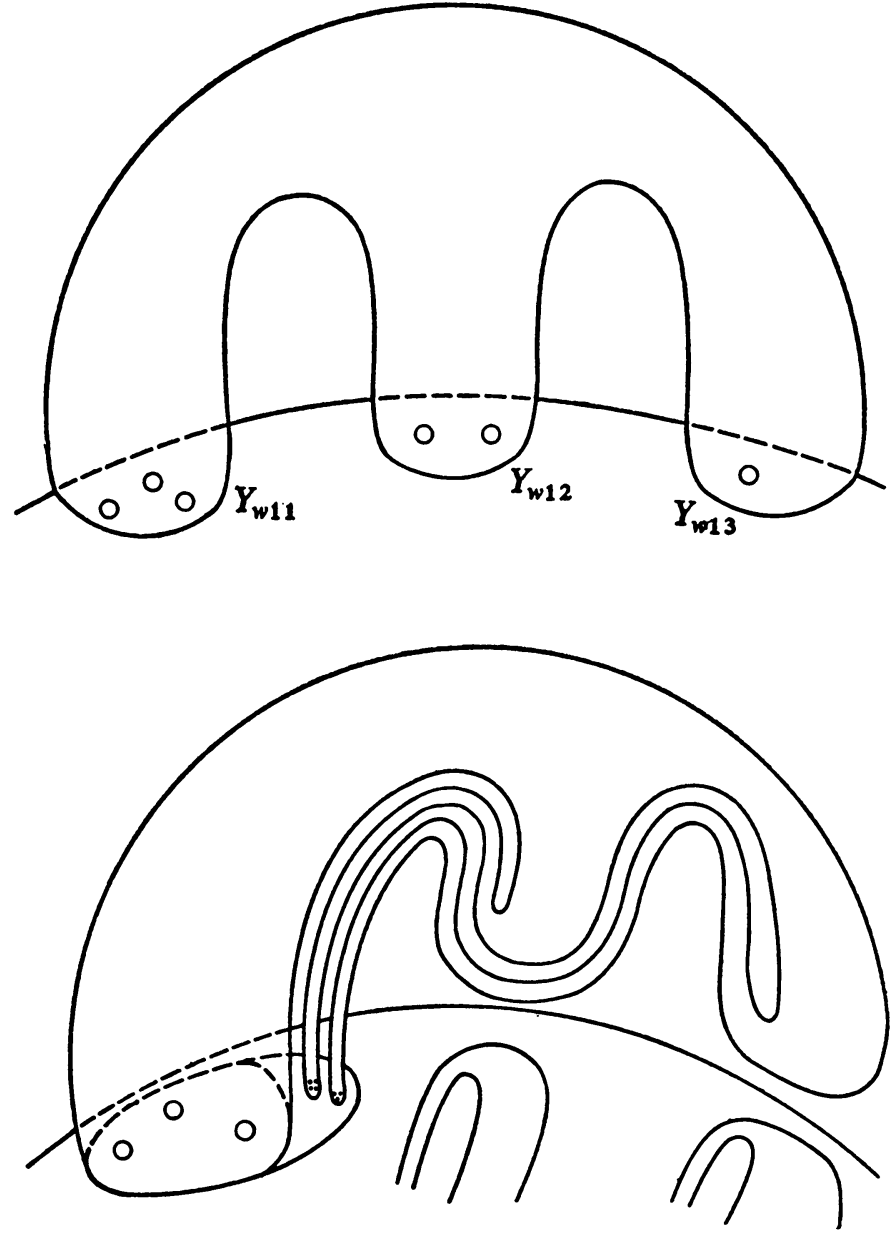

Figure 5 
in Case 1 using $T_{w 11}^{\prime \prime}$ in place of $T_{w 1}^{\prime \prime}$ gives the existence of a homeomorphism $f_{1}$ with the desired properties. This concludes the proof of Step I.

It follows that there exist homeomorphisms $f_{2}, \cdots$, and $f_{t(w)}$ satisfying the following: (1) $f_{t(w)} \cdots f_{2} f_{1}$ is a homeomorphism from $M$ into $E^{3}$, (2) $f_{t(w)} \cdots f_{1}[M]$ satisfies conditions (1)-(7) (3) If $V^{\prime \prime}$ is the large component of $f_{t(w)} \cdots f_{1}[M]-S^{\prime}$ then $f_{t(w)} \cdots f_{1}[M]-V^{\prime \prime}$ has at most $p$ components, and (4) $f_{t(w)} \cdots f_{1}[M]$ is contained in

$$
\left(M-\mathrm{Cl}\left[\bigcup_{k=1}^{t(w)} X_{k}^{w}\right]\right) \cup \bigcup_{k=1}^{t(w)} T_{w k}^{\prime \prime} \text {. }
$$

Step II. In Step II it is proved that there exists a homeomorphism $h_{i}$ from $M$ into $E^{3}$ such that $h_{i}\left[F_{i}\right] \subset \operatorname{Int} S^{\prime}, h_{t}[M]$ satisfies conditions (1)-(7), and if $V^{\prime}$ is the large component of $h_{i}[M]-S^{\prime}$ then $K_{i}[M]-V^{\prime}$ has at most $p-1$ components. This will conclude the proof of Part $\mathrm{I}$.

Proof of STEP II. Using an argument similar to the one given in Step I, it can be shown that there exists a homeomorphism $f_{t(w)+1}$ from $f_{t(w)} \cdots f_{1}[M]$ into $E^{3}$ with the following properties: (1) $f_{t(w)+1} \cdots f_{1}[M]$ satisfies conditions (1)-(7), (2) Each component of $f_{t(w)} \cdots f_{1}[M]-K_{j}$ that intersects $X_{1}^{w-1}$ is mapped into $\left(M-\left[\bigcup_{k=1}^{t(w)} X_{k}^{w} \cup X_{1}^{w-1}\right]\right) \cup\left[\bigcup_{k=1}^{t(w-1)} T_{(w-1) k}^{\prime \prime} \cup T_{w}^{\prime \prime}\right]$, and (3) If $V^{\prime \prime \prime}$ is the large component of $f_{t(w)+1} \cdots f_{1}[M]-S^{\prime}$ then $f_{t(w)+1} \cdots f_{1}[M]-V^{\prime \prime \prime}$ has at most $p$ components. After $t(1)+t(2)+\cdots+t(w-1)-1$ additional repetitions of this argument the homeomorphism $h_{i}$ is obtained. Since $h_{i}\left[F_{i}\right]$ is contained in the union of $T_{1}^{\prime \prime}$ and a small neighborhood of $V$, it follows that $h_{i}\left[F_{i}\right]$ is contained in Int $\mathrm{S}^{\prime}$.

PART II. In this part, it is supposed that for each integer $j$ less than or equal to $p$, there does not exist an integer $i$ such that $F_{\imath} \cap \mathrm{Cl} V$ is contained in $K_{j}$. It is shown that there exist an integer $k, 1 \leqq k \leqq m$, and a homeomorphism $h_{1}$ from $M$ into $E^{3}$ such that (1) $h_{1}[M]$ satisfies conditions (1)-(7), (2) If $V^{\prime \prime}$ denotes the large component of $h_{1}[M]-S^{\prime}$ then $h_{1}[M]-V^{\prime \prime}$ has at most $p$ components, and (3) $h_{1}\left[F_{1}\right] \cup \mathrm{Cl} V^{\prime \prime}$ is contained in $K_{k}$.

Proof of Part II. An argument similar to the one given in the proof of Part I, Step I, Case 2 shows that there exists a homeomorphism $h_{1}$ from $M$ into $E^{3}$ such that $h_{1}$ has the desired properties

PART III. In this part, it is shown that there is a homeomorphism $h$ which satisfies the conclusion of Theorem 1.

Proof of Part III. Assume the $F_{i}$ 's are indexed so that there exists an integer $u, 1 \leqq u \leqq p$, with the property that if $i<u$ then there exists an integer $k, 1 \leqq k \leqq m$, such that $F_{i} \cap \mathrm{Cl} V$ is contained in $K_{k}$ and if $i \geqq u$ then there does not exist an integer $k, 1 \leqq k \leqq m$, such that $F_{i} \cap \mathrm{Cl} V \subset K_{k}$. If $u$ is greater than 1 , apply Part I to $F_{1}$ and obtain a homeomorphism $h_{1}$ from $M$ into $E^{3}$ that maps $F_{1}$ into Int $S^{\prime}$. Then apply Part I to $h_{1}\left[F_{2}\right]$ obtaining $h_{2}$. Continue this process to obtain homeomorphisms $h_{3}, \cdots$, and $h_{u-1}$. Then by applying Part II to $h_{u-1} \cdots h_{1}\left[F_{u}\right]$, a homeo- 
morphism $h_{u}$ is obtained, and the results of Part I are then applied to $h_{u} \cdots h_{1}\left[F_{u}\right]$ to obtain $h_{u+1}$. Then by using Part I or Part II, depending upon $h_{u+1} \cdots h_{1}\left[F_{u+1}\right]$, a homeomorphism $h_{u+2}$ is obtained. Continue this process. Let $h$ denote the composition of the $h_{i}$ 's. Clearly $h$ satisfies conditions (1)-(8). Condition (9) follows since for each $i$ less than or equal to $p$, the diameter of $F_{i}$ is less than $5 e^{\prime \prime}$, the diameter of $h\left[F_{i}\right]$ is less than $5 e^{\prime \prime}$, and $h$ moves no point of $V$ more than $e^{\prime \prime}$. This concludes the proof of Theorem 1 .

THEOREM 2. If $C$ is a crumpled cube in $S^{3}$ and $e$ is a positive number, then there exists a homeomorphism $h$ from $C$ into $S^{3}$ such that the closure of $S^{3}-h[C]$ is a 3-cell and if $x$ belongs to $C$ then $d(x, h(x))$ is less than $e$.

Proof. Let $f$ be a positive sequence such that the series whose sequence of terms is $f$ converges to a number less than $e$, and let $C_{1}, C_{2}, \cdots$ be a sequence of closed sets such that for each $i, C_{i} \subset \operatorname{Int} C_{i+1}$, and $\bigcup_{i=1}^{\infty} C_{i}=\operatorname{Int} C$. Let $S$ denote the boundary of $C$.

By Theorem 1 there exists a polyhedral 2-sphere $S_{1}$ and a homeomorphism $h_{1}$ from $C$ into $S^{3}$ such that $S_{1}$ is homeomorphically within $f(1)$ of $S, h_{1}[C] \subset \operatorname{Int} S_{1}$ if $x \in C$ then $d\left(x, h_{1}(x)\right)<f(1)$, and if $x \in C_{1}$ then $h_{1}(x)=x$.

Let $e_{2}$ denote $(1 / 3) \min \left\{f(2), d\left(S_{1}, h_{1}[S]\right)\right\}$. Then by Theorem 1 there exists a polyhedral 2-sphere $S_{2}$ and a homeomorphism $h_{2}^{\prime}$ from $h_{1}[C]$ into $S^{3}$ such that $S_{2}$ is homeomorphically within $e_{2}$ of $h_{1}[S], h_{2}^{\prime} h_{1}[C] \subset \operatorname{Int} S_{2}$, if $x \in h_{1}\left[C_{2}\right]$ then $h_{2}^{\prime}(x)=x$, and if $x \in h_{1}[C]$ then $d\left(x, h_{2}^{\prime}(x)\right)<e_{2}$. Let $h_{2}$ be $h_{2}^{\prime} h_{1}$. It follows that $h_{2}$ maps $C$ Int $S_{2}$, if $x \in C$ then $d\left(x, h_{2}(x)\right)<f(1)+f(2)$, and $S_{2} \subset \operatorname{Int} S_{1}$.

Proceeding by induction, there exists a 2-sphere $S_{n}$ and a homeomorphism $h_{n}^{\prime}$ from $h_{n-1}[C]$ into $S^{3}$ such that $S_{n}$ is homeomorphically within $f(n)$ of $h_{n-1}[S]$, $h_{n}^{\prime} h_{n-1}[C] \subset \operatorname{Int} S_{n}$, if $x \in h_{n-1}\left[C_{n}\right]$ then $h_{n}^{\prime}(x)=x, S_{n} \subset \operatorname{Int} S_{n-1}$, and if $x \in h_{n-1}[C]$ then $d\left(x, h_{n}^{\prime}(x)\right)<f(n)$. Let $h_{n}$ be $h_{n}^{\prime} h_{n-1}$.

The sequence $h_{1}, h_{2}, \cdots$ is uniformly convergent. Let $h$ be the limit of $h_{1}, h_{2}, \cdots$. It follows that $h$ is uniformly continuous and into $S^{3}$.

It is to be proved that $h$ is one-to-one.

LEMMA 1. The restriction of $h$ to the interior of $C$ is one-to-one.

Proof. Suppose $x$ and $y$ are distinct points in Int $C$. There exists a positive integer $j$ such that $x$ and $y$ are in $C_{j}$. Hence for each integer $k \geqq j, h_{k}(x)=h_{j}(x)$, $h_{k}(y)=h_{j}(y)$, and $h_{j}(x) \neq h_{j}(y)$. Then $h(x) \neq h(y)$.

LEMMA 2. For each $x$ in $h[\operatorname{Int} C], \lim h_{i}^{-1}(x)$ exists and is equal to $h^{-1}(x)$.

Proof. $\operatorname{Sup}\left\{d\left(h_{i}^{-1}(x), h_{i+1}^{-1}(x)\right): x\right.$ in $\left.h[C]\right\} \leqq f(i)$. Hence the sequence $h_{1}^{-1}, h_{2}^{-1}, \cdots$ is uniformly convergent on $h[C]$ and therefore, for each $x$ in $h[C]$, $\lim h_{i}^{-1}(x)$ exists. Suppose $x$ belongs to $h[\operatorname{Int} C]$. By Lemma 1 there is one and only one point $y$ in Int $C$ such that $h(y)=x$ or $y=h^{-1}(x)$. There exists a positive integer $j$ such that $y \in C_{j}$, hence $h(y)=h_{j}(y)$ and $\lim h_{i}^{-1}(x)=h_{j}^{-1}(x)=y$. 
LEMMA 3. The function $h$ is a homeomorphism from $C$.

Proof. It suffices to show $h$ is one-to-one from $C$. Suppose $h$ is not one-to-one. Then there exist distinct points $x$ and $y$ in $C$ such that $h(x)=h(y)$. Let $x_{1}, x_{2}, \cdots$, and $y_{1}, y_{2}, \cdots$ be sequences such that for each $i, x_{i}$ and $y_{i}$ belong to Int $C$, the sequence $\left\{x_{i}\right\}$ converges to $x$, and the sequence $\left\{y_{i}\right\}$ converges to $y$. Then the sequence $\left\{h\left(x_{i}\right)\right\}$ converges to $h(x)$ and the sequence $\left\{h\left(y_{i}\right)\right\}$ converges to $h(y)$ which is equal to $h(x)$. Let $e^{\prime}$ be a positive number less than $d(x, y) / 2$. Since $h$ is uniformly continuous on $h$ [Int $C$ ], there is a positive number $r$ such that if $d(p, q)<r$, then $d\left(h^{-1}(p), h^{-1}(q)\right)<e^{\prime}$. But there exists a positive integer $i$ such that $d\left(h\left(x_{i}\right), h\left(y_{i}\right)\right)<r$ and $d\left(x_{i}, y_{i}\right)>e^{\prime}$. This is a contradiction.

LEMMA 4. If $x$ belongs to $C$ then $d(x, h(x))$ is less than $e$.

Proof. $d(x, h(x)) \leqq \lim _{n \rightarrow \infty}\left(d\left(x, h_{1}(x)\right)+d\left(h_{1}(x), h_{2}(x)\right)+\cdots+d\left(h_{n-1}(x), h_{n}(x)\right)\right)$ $\leqq \sum_{i=1}^{\infty} f(i)<e$.

LEMMA 5. The closure of $S^{3}-h[C]$ is a 3-cell.

Proof. For each positive integer $i, S_{i}$ is homeomorphically within $f(i)$ of $h_{i-1}[S]$ and $h_{i-1}[S]$ is homeomorphically within $\sum_{j=i}^{\infty} f(j)$ of $h[S]$. Therefore $S_{i}$ is homeomorphically within $f(i)+\sum_{j=i}^{\infty} f(j)$ of $h[S]$. For each positive integer $i, S_{i}$ is a polyhedral 2-sphere in Ext $h[C]$. It follows from Theorem 2.1 of [5] that the closure of $S^{3}-h[C]$ is a 3 -cell. This concludes the proof of Theorem 2 .

3. Crumpled cubes as decomposition spaces. The following definitions and terminology will be used in $\S 3$. If $r$ is a positive number, $B(r)$ will denote the ball in $E^{3}$ with center at the origin and radius $r$. If $S$ and $T$ are 2-spheres in $E^{3}$ and $S \subset \operatorname{Int} T$, then $[S, T]$ will denote $\mathrm{Cl}(\operatorname{Int} T-\operatorname{Int} S)$. Suppose $r$ and $s$ are positive numbers and $r<s$. A cellular subdivision $\left\{E_{1}, \cdots, E_{q}\right\}$ of $[\mathrm{Bd} B(r), \mathrm{Bd} B(s)]$ is a canonical subdivision of $[\mathrm{Bd} B(r), \mathrm{Bd} B(s)]$ if and only if there exist disks $D_{1}, \cdots$, and $D_{q}$ on $\operatorname{Bd} B(r)$ such that (1) for each $i$, there exists a $j$ such that if $p$ is in $E_{i}$, then there exists a point $t$ in $D_{j}$ and a real number $c$ such that $p=c t$, (2) if $i \neq j$ and $D_{i} \cap D_{j}$ is nonempty, then $D_{i} \cap D_{j} \subset \operatorname{Bd} D_{i} \cap \operatorname{Bd} D_{j}$, (3) for each $i$, $E_{i} \cap B(r)$ is nonempty, and (4) for each $i$ and $j$, the diameter of $D_{i}$ is at most twice the diameter of $D_{j}$. Suppose $S_{1}$ and $S_{2}$ are tame 2-spheres in $E^{3}$ and $S_{2}$ is contained in Int $S_{1}$. A cellular subdivision $\left\{E_{1}, \cdots, E_{q}\right\}$ of $\left[S_{2}, S_{1}\right]$ is an $A$-subdivision of $\left[S_{2}, S_{1}\right]$ if and only if there exists a homeomorphism $h$ from $\left[S_{2}, S_{1}\right]$ onto $[\mathrm{Bd} B(1), \mathrm{Bd} B(2)]$ such that $\left\{h\left[E_{1}\right], \cdots, h\left[E_{q}\right]\right\}$ is a canonical subdivision of $[\mathrm{Bd} B(1), \mathrm{Bd} B(2)]$. If $X$ is a topological space and $G$ is an upper semi-continuous decomposition of $X$, then $X / G$ will denote the decomposition space associated with $G$.

THEOREM 3. If $C$ is a crumpled cube and $\mathrm{Cl}\left(S^{3}-C\right)$ is a 3-cell, then there exists an upper semi-continuous decomposition $G$ of $B(1)$ such that the intersection 
of each nondegenerate element of $G$ with the boundary of $B(1)$ is a one-point set, each nondegenerate element of $G$ is point-like, and $B(1) / G$ is homeomorphic to $C$.

Proof. Let $S$ denote the boundary of $C$, and let $e_{1}$ denote $\min \{1,1 / 10$ diameter of $C\}$. There exists a polyhedral 2-sphere $S_{1}$ in Ext $S$ such that $S_{1}$ is homeomorphically within $e_{1}$ of $S$. Now let $e_{11}$ be $(1 / 2) d\left(C, S_{1}\right)$. By the Side Approximation Theorem there exists a polyhedral 2-sphere $S_{11}$ with the following properties: (1) $S_{11}$ is homeomorphically within $e_{11}$ of $S$, (2) there exist disjoint disks $D_{11}, \cdots$, and $D_{1 n(1)}$ on $S$, each of diameter less than $e_{11}$, such that $\mathrm{Cl}\left(S-\bigcup_{i=1}^{n(1)} D_{1 i}\right)$ is contained in Ext $S_{11}$, and (3) there exist disjoint disks $K_{11}, \cdots$, and $K_{1 m(1)}$ on $S_{11}$, each of diameter less than $e_{11}$, such that $\mathrm{Cl}\left(S_{11}-\bigcup_{i=1}^{m(1)} K_{1 i}\right)$ is contained in Int $S$. Let $e_{12}$ denote

$$
\begin{gathered}
(1 / 3) \min \left\{\min \left\{d\left(D_{1 i}, D_{1 j}\right): i \neq j, 1 \leqq i \leqq n(1), \text { and } i \leqq j \leqq n(1)\right\},\right. \\
\min \left\{d\left(K_{1 i}, K_{1 j}\right): i \neq j, 1 \leqq i \leqq m(1), \text { and } 1 \leqq j \leqq m(1)\right\}, \\
\left.\min \left\{d\left(D_{1 i}, S_{11}-\bigcup_{j=1}^{m(1)} K_{1 j}\right): 1 \leqq i \leqq n(1)\right\}\right\}
\end{gathered}
$$

Let $T_{1}$ be a triangulation of $S^{3}$ with mesh less than $e_{12}$, let $N$ be the union of all 3-simplexes of $T$ which intersect $\left[S, S_{1}\right]$, and let $M_{11}$ be the union of all 3-simplexes in the 2nd barycentric subdivision of $T$ which intersect $N$. Then $M_{11}$ is a polyhedral 3-manifold with boundary and it can be assumed that $M_{11}$ and $S_{11}$ are in relative general position.

Let $M_{1}$ denote the union of $M_{11}$ and $\left[S_{11}, S_{1}\right]$. Now $M_{1}-\left[S_{11}, S_{1}\right]$ has a finite number of components, and let $F_{11}, \cdots$, and $F_{1 r(1)}$ be the components of $\mathrm{Cl}\left(M_{1}-\left[S_{11}, S_{1}\right]\right)$. It follows from the Side Approximation Theorem that for each $i, F_{1 i}$ has diameter less than $4 e_{1}+2 e_{12}$ which is less than $5 e_{1}$.

There exists an $A$-subdivision $\left\{E_{11}, \cdots, E_{1 q(1)}\right\}$ of $\left[S_{11}, S_{1}\right]$ such that (1) for each $i, i \leqq n(1)$, there exists a positive integer $j$ such that $\operatorname{Bd} D_{1 i} \subset \operatorname{Int} E_{1 j}$, and (2) for each $i, i \leqq q(1)$, the diameter of $E_{1 i}$ is less than $5 e_{1}$. Let $A_{1 i}^{*}$ and $A_{1 i}$, $i \leqq q(1)$, be defined as follows: $A_{1 i}^{*}=\bigcup\left\{X: X \in\left\{E_{11}, \cdots, E_{1 q(1)}\right\}\right.$ or $X \in\left\{F_{11}, \cdots, F_{1 r(1)}\right\}$ and $\left.d\left(X, E_{1 i}\right)<e_{1}\right\}$ and $A_{1 i}$ is the component of $A_{1 i}^{*}$ that contains $E_{1 i}$. Note that for each $p$ in $S$, there exists an integer $j$ such that $p$ is in $A_{1 j}$. For each $p$ in $S$, denote $\bigcup\left\{A_{1 i}: p \in A_{1 i}\right\}$ by $A_{p 1}$. Now for each $i$, the diameter, of $A_{1 i}$ is at most $17 e_{1}$, hence for each $p$, the diameter of $A_{p 1}$ is at most $34 e_{1}$.

Then there exists a homeomorphism $h_{1}$ from $S_{1} \cup$ Int $S_{1}$ onto $B(1.1)$ with the following properties: (1) $h_{1}\left[\left[S_{11}, S_{1}\right]\right]=[\mathrm{Bd} B(.9), \mathrm{Bd} B(1.1)]$, (2) $h_{1}[C]$ is contained in Int $B(1.01)$, and (3) $\left\{h_{1}\left[E_{11}\right], \cdots, h_{1}\left[E_{1 q(1)}\right]\right\}$ is a canonical subdivision of $[\mathrm{Bd} B(.9), \mathrm{Bd} B(1.1)]$ into $q(1)$ cells.

Now $M_{1}$ is a 3-manifold-with-boundary and $S$ is a compact subset in Int $M_{1}$. It follows that there exists a positive number $e_{13}$ such that, if $Q$ is a set with diameter less than $34 e_{13}$ and the intersection of $Q$ with $S$ exists, then there exists a cell $R$ such that $Q \subset \operatorname{Int} R \subset R \subset \operatorname{Int} M_{1}$. 
Let $e_{2}$ denote $\min \left\{d\left(S, \mathrm{Bd} M_{1}\right), e_{13}, 1 / 4\right\}$. There exists a polyhedral 2-sphere $S_{2}$ in Ext $S$ such that $S_{2}$ is homeomorphically within $e_{2}$ of $S$. Let $e_{21}$ be $(1 / 3) \min \left\{d\left(S, S_{2}\right), \min \left\{d\left(\operatorname{Bd} D_{1 i}, \operatorname{Bd} E_{1 j}\right): \operatorname{Bd} D_{1 i} \subset \operatorname{Int} E_{1 j}\right\}\right\}$. By the Side Approximation Theorem there exists a polyhedral 2-sphere $S_{22}$ such that (1) $S_{22}$ is homeomorphically within $e_{21}$ of $S$, (2) there exist disjoint disks $D_{21}, \cdots$, and $D_{2 n(2)}$ on $S$, each of diameter less than $e_{21}$, such that $\mathrm{Cl}\left(S-\bigcup_{i=1}^{n(2)} D_{2 i}\right) \subset \operatorname{Ext} S_{22}$, and (3) there exists disjoint disks $K_{21}, \cdots$, and $K_{2 m(2)}$ on $S_{22}$, each of diameter less than $e_{21}$, such that $\mathrm{Cl}\left(S_{22}-\bigcup_{i=1}^{m(2)} K_{2 i}\right) \subset$ Int $S$. Let $e_{22}$ denote

$(1 / 3) \min \left\{\min \left\{d\left(D_{2 i}, D_{2 j}\right): i \neq j, 1 \leqq i \leqq n(2)\right.\right.$, and $\left.1 \leqq j \leqq n(2)\right\}$,

$$
\begin{gathered}
\min \left\{d\left(K_{2 i}, K_{2 j}\right): i \neq j, 1 \leqq i \leqq m(2), \text { and } 1 \leqq j \leqq m(2)\right\}, \\
\left.\min \left\{d\left(D_{2 i}, S_{22}-\bigcup_{j=1}^{m(2)} K_{2 j}\right): 1 \leqq i \leqq n(2)\right\}\right\} .
\end{gathered}
$$

If $M_{22}^{\prime}=\left\{z: z \in S_{2} \cup \operatorname{Int} S_{2}\right.$ and $d(z$, Ext $\left.S) \leqq e_{22}\right\}$ then in the same way as $M_{11}$ was obtained, a polyhedral 3-manifold-with-boundary $M_{22}$ exists such that $\left[S, S_{2}\right] \subset \operatorname{Int} M_{22}$ and $M_{22} \subset M_{22}^{\prime}$. It can be assumed that $M_{22}$ and $S_{22}$ are in relative general position. Let $M_{2}$ denote the union of $M_{22}$ and $\left[S_{22}, S_{2}\right]$. Now $M_{2}-\left[S_{22}, S_{2}\right]$ has a finite number of components, and let $F_{21}, \cdots$, and $F_{2 r(2)}$ be the components of $\mathrm{Cl}\left(M_{2}-\left[S_{22}, S_{2}\right]\right)$. It follows that for each $i$, the diameter of $F_{2 i}$ is at most $5 e_{2}$. It follows from the definition of $M_{2}$ that $S \subset \operatorname{Int} M_{2} \subset M_{2} \subset$ Int $M_{1}$ and that $\left\{x: x \in C\right.$ and $\left.d(x, S)>e_{22}\right\}$ is disjoint from Int $M_{2}$.

There exists an $A$-subdivision $\left\{E_{21}, \cdots, E_{2 q(2)}\right\}$ of $\left[S_{22}, S_{2}\right]$ such that (1) for each $i, 1 \leqq i \leqq n(2)$, there exists a positive integer $j$ such that $\operatorname{Bd} D_{2 i} \subset \operatorname{Int} E_{2 j}$, (2) for each $i, 1 \leqq i \leqq q(2)$, the diameter of $E_{2 i}$ is less than $5 e_{2}$, and (3) $q(2) \geqq 4 q(1)$. Let $A_{2 i}^{*}$ and $A_{2 i}$ be defined as follows: $A_{2 i}^{*}=\bigcup\left\{X: X \in\left\{E_{21}, \cdots, E_{2 q(2)}\right\}\right.$ or $X \in\left\{F_{21}, \cdots, F_{2 r(2)}\right\}$ and $\left.d\left(X, E_{2 i}\right)<e_{2}\right\}$ and $A_{2 i}$ is the component of $A_{2 i}^{*}$ that contains $E_{2 i}$. For each $p$ in $S$, there exists an integer $j$ such that $p$ is in $A_{2 j}$. For each $p$ in $S$, denote $\bigcup\left\{A_{2 i}: p \in A_{2 i}\right\}$ by $A_{p 2}$. Note that for each $p$ in $S$, the diameter of $A_{p 2}$ is at most $34 e_{2}$. It follows that for each $p$ in $S, A_{p 2} \subset \operatorname{Int} A_{p 1}$. Furthermore, if $p \in\left[S_{11}, S_{1}\right]$ and $Z_{p 1}$ is the component of $A_{p 1} \cap\left[S_{11}, S_{1}\right]$ that contains $p$, then $\left[S_{22}, S_{2}\right] \cap A_{p 2}$ is contained in $Z_{p 1}$ and for each $p$ in $S$, there exists a 3-cell $C_{p 2}$ such that $A_{p 2} \subset \operatorname{Int} C_{p 2} \subset C_{p 2} \subset \operatorname{Int} A_{p 1}$.

Now $S_{2} \cap\left\{\bigcup_{i=1}^{q(1)} \mathrm{Bd} E_{1 i}\right\}$ exists and has one and only one component with diameter greater than $1 / 2$ the diameter of $C$. Denote this component by $W_{1}$. It follows that for each $i, 1 \leqq i \leqq q(1)$, the intersection of $W_{1}$ and $E_{1 i}$ exists.

There exists a homeomorphism $h_{2}$ from $S_{2} \cup \operatorname{Int} S_{2}$ onto $B(1.01)$ with the following properties: (1) $h_{2}\left[\left[S_{22}, S_{2}\right]\right]=[\mathrm{Bd} B(.99), \mathrm{Bd} B(1.01)],(2) h_{2}[C]$ is contained in Int $B(1.001)$, (3) if $x \in W_{1}$ then $h_{2}(x)$ is equal to the radial projection of $h_{1}(x)$ onto $\mathrm{Bd} B(1.01)$, (4) if $x$ is in $C-M_{2}$, then $h_{2}(x)=h_{1}(x)$, (5) $h_{2}$ differs from $h_{1}$ only at points close to $\left[S_{22}, S_{2}\right]$, and (6) $\left\{h_{2}\left[E_{21}\right], \cdots, h_{2}\left[E_{2 q(2)}\right]\right\}$ is a canonical subdivision of $[\mathrm{Bd} B(.99), \mathrm{Bd} B(1.01)]$. 
The process is continued by induction. Hence for each positive $i, e_{i}, e_{i 1}, e_{i 2}$, $e_{(i-1) 3}, S_{i}, S_{i i}, D_{i 1}, \cdots, D_{i n(i)}, K_{i 1}, \cdots, K_{i m(i)}, M_{i i}, M_{i}, F_{i 1}, \cdots, F_{i r(i)}, E_{i 1}, \cdots, E_{i q(i)}$, $A_{i 1}^{*}, \cdots, A_{i q(i)}^{*}, A_{i 1}, \cdots, A_{i q(i)}, A_{p i}, Z_{p(i-1)}, C_{p i}, W_{i}$, and $h_{i}$ are defined inductively as follows: (1) Since $S$ is contained in Int $M_{i-1}$, there exists a positive number $e_{(i-1) 3}$ such that if $Q$ is a set of diameter less than $34 e_{(i-1) 3}$ and the intersection of $Q$ with $S$ exists, then there exists a 3-cell $R$ such that $Q \subset \operatorname{Int} R \subset R \subset \operatorname{Int} M_{i-1}$. (2) Let $e_{i}$ be $(1 / 3) \min \left\{e_{(i-1) 3}, 2^{-i}, d\left(C, \mathrm{Bd} M_{i-1}\right)\right\}$. (3) $S_{i}$ is a polyhedral 2-sphere in Ext $S$ such that $S_{i}$ is homeomorphically within $e_{i}$ of $S$. (4) Let $e_{i 1}$ be $(1 / 3) \min \left\{d\left(C, S_{i}\right), \min \left\{d\left(\operatorname{Bd} D_{(i-1) j}, E_{(i-1) k}\right): \operatorname{Bd} D_{(i-1),} \subset \operatorname{Int} E_{(i-1) k}\right\}\right\}$. (5) $S_{i i}$ is a polyhedral 2-sphere that is homeomorphically within $e_{i 1}$ of $S$, such that there exist disjoint disks $D_{i 1}, \cdots$, and $D_{i n(i)}$ on $S$, each of diameter less than $e_{i 1}$, with the property that $\mathrm{Cl}\left(S-\bigcup_{j=1}^{i(i)} D_{i j}\right)$ is contained in Ext $S_{i i}$, and there exist disjoint disks $K_{i 1}, \cdots$, and $K_{i m(i)}$ on $S_{i i}$, each of diameter less than $e_{i 1}$, with the property that $\mathrm{Cl}\left(S_{i i}-\bigcup_{j=1}^{m(i)} K_{i j}\right)$ is contained in Int $S$. Such a 2 -sphere exists by Bing's Side Approximation Theorem. (6) Let $e_{i 2}$ be

$$
\begin{gathered}
(1 / 3) \min \left\{\min \left\{d\left(D_{i u}, D_{i v}\right): u \neq v, 1 \leqq u \leqq n(i), \text { and } 1 \leqq v \leqq n(i)\right\},\right. \\
\min \left\{d\left(K_{i u}, K_{i v}\right): u \neq v, 1 \leqq u \leqq m(i), \text { and } 1 \leqq v \leqq m(i)\right\}, \\
\left.\min \left\{d\left(D_{i u}, S_{i i}-\bigcup_{j=1}^{m(i)} K_{i j}\right): 1 \leqq u \leqq n(i)\right\}\right\} .
\end{gathered}
$$

(7) Let $M_{i i}$ be $\left\{x: x \in S_{i} \cup\right.$ Int $S_{i}$ and $d(x$, Ext $\left.S) \leqq e_{i 2}\right\}$ and let $M_{i i}$ be a polyhedral 3-manifold-with-boundary such that $\left[S, S_{i}\right] \subset \operatorname{Int} M_{i i}$ and $M_{i i} \subset M_{i i}^{\prime}$. It is assumed that $M_{i i}$ and $S_{i i}$ are in relative general position. (8) Let $M_{i}$ be the union of $M_{i i}$ and $\left[S_{i i}, S_{i}\right]$. (9) The closure of $M_{i}-\left[S_{i i}, S_{i}\right]$ has a finite number of components, and let $F_{i 1}, \cdots$, and $F_{i r(i)}$ be the components of $\mathrm{Cl}\left(M_{i}-\left[S_{i i}, S_{i}\right]\right) .(10)\left\{E_{i 1}, \cdots, E_{i q(i)}\right\}$ is an $A$-subdivision of $\left[S_{i i}, S_{i}\right]$ such that (a) for each $j, 1 \leqq j \leqq n(i)$, there exists a $k$ such that $\operatorname{Bd} D_{i j} \subset \operatorname{Int} E_{i k}$, (b) for each $j, 1 \leqq j \leqq q(i)$, the diameter of $E_{i j}$ is less than $5 e_{i}$, and (c) $q(i) \geqq q(i-1)$. (11) For each $j, 1 \leqq j \leqq q(i), A_{i j}^{*}$ and $A_{i j}$ are defined as follows: $A_{i j}^{*}=\bigcup\left\{x: x \in\left\{E_{i 1}, \cdots, E_{i q(i)}\right\}\right.$ or $x \in\left\{F_{i 1}, \cdots, F_{i r(i)}\right\}$ and $\left.d\left(x, E_{i j}\right)<e_{i}\right\}$, and $A_{i j}$ is the component of $A_{i j}^{*}$ that contains $E_{i j}$. (12) For each $p$ in $S$, let $A_{p i}$ be $\bigcup\left\{A_{i j}: p \in A_{i j}\right\}$. (13) For each $p$ in $S$, if $p$ is in $\left[S_{i i}, S_{i}\right]$ then $Z_{p(i-1)}$ is the component of $A_{p i} \cap\left[S_{i i}, S_{i}\right]$ that contains $p$. (14) For each $p$ in $S$, $C_{p i}$ is a 3-cell that contains $A_{p i}$ in its interior and is contained in the interior of $M_{i-1}$. (15) $W_{i-1}$ is the component of $S_{i} \cap\left\{\bigcup_{j=1}^{q(i-1)} \mathrm{Bd} E_{(i-1) j}\right\}$ with diameter greater than $(1 / 2)$ diam $C$. (16) $h_{i}$ is a homeomorphism from $S_{i} \cup \operatorname{Int} S_{i}$ onto $B\left(1+10^{-i}\right)$ such that (a) $h_{i}\left[\left[S_{i i}, S_{i}\right]\right]=\left[\mathrm{Bd} B\left(1-10^{-i}\right), \quad \mathrm{Bd} B\left(1+10^{-i}\right)\right]$, (b) $h_{i}[C] \subset$ Int $B\left(1-10^{-i-1}\right)$, (c) if $x \in W_{i-1}$ then $h_{i}(x)$ is equal to the radial projection of $h_{i-1}(x)$ onto $\mathrm{Bd} B\left(1+10^{-i}\right)$, (d) if $x$ is in $C-M_{i}$ then $h_{i}(x)=h_{i-1}(x)$, (e) $h_{i}$ differs from $h_{i-1}$ only at points close to $\left[S_{i i}, S_{i}\right]$, and (f) $\left\{h_{i}\left[E_{i 1}\right], \cdots, h_{i}\left[E_{i q(i)}\right]\right\}$ is a canonical subdivision of $\left[\mathrm{Bd} B\left(1-10^{-i}\right), \mathrm{Bd} B\left(1+10^{-i}\right)\right]$.

It follows that $B(1)=\bigcap_{i=1}^{\infty} h_{i}\left[S_{i} \cup \operatorname{Int} S_{i}\right]$. Let $G$ be the following decomposition of $B(1): G=\{g:$ either (1) for some point $p$ of $B(1)$ there exists a positive 
integer $n$ such that $p \notin h_{n}\left[M_{i i}\right]$ and $g=\{p\}$, or (2) there exists a $q$ in $S$ such that $\left.g=\bigcap_{i=1}^{\infty} h_{i}\left[A_{q i}\right]\right\}$. Then $G$ is an upper semi-continuous decomposition of $B(1)$. It follows from the way the $A_{p i}$ 's and the $h_{i}$ 's are defined that the intersection of each nondegenerate element of $G$ with the boundary of $B(1)$ is a one-point set.

If $g$ is a nondegenerate element of $G$, then there exists a $q$ in $S$ such that $g=\bigcap_{i=1}^{\infty} h_{i}\left[A_{q i}\right]$. But $\bigcap_{i=1}^{\infty} h_{i}\left[A_{q i}\right]$ is equal to $\bigcap_{i=1}^{\infty} h_{i}\left[C_{q i}\right]$. Hence $g$ is point-like.

Define a function $h$ from $C$ into $B(1) / G$ as follows: If $x \in \operatorname{Int} C, h(x)=\left\{\lim h_{i}(x)\right\}$ and if $x \in S, h(x)=\bigcap_{i=1}^{\infty} h_{i}\left[A_{x i}\right]$. Then $h$ is one-to-one. If not, there exist points $p$ and $q$ in $C$ such that $p \neq q$ and $h(p)=h(q)$. Clearly, neither $p$ nor $q$ can be in Int $C$. If $p$ and $q$ are in $S$ then there exists an integer $n$ such that $A_{p n}$ and $A_{q n}$ are disjoint, and hence $h_{n}\left[A_{p n}\right]$ and $h_{n}\left[A_{q n}\right]$ are disjoint. This is a contradiction. Clearly $h$ is onto $B(1) / G$ and $h$ is a homeomorphism on Int $C$. To show $h$ is a homeomorphism it suffices to show $h$ is continuous at each point of $S$. Suppose $p$ is in $S,\left\{a_{i}\right\}$ is a sequence in $C$, and $\left\{a_{i}\right\}$ converges to $p$. If $U$ is an open set in $B(1) / G$ that contains $h(p)$, then there exists a positive integer $k$ such that $h_{k}\left[A_{p k}\right]$ is contained in $U$. Since $\left\{a_{i}\right\}$ converges to $p$, all but finitely many of the $a_{i}$ 's belong to $A_{p i}$. Therefore all but finitely many of the $h_{i}\left(a_{i}\right)$ 's belong to $h_{i}\left[A_{p i}\right]$. By the way the $h_{i}$ 's are constructed, if $j \geqq i$ and $h_{i}\left(a_{i}\right) \in h_{i}\left[A_{p i}\right]$, then $h_{j}\left(a_{i}\right) \in h_{i}\left[A_{p i}\right]$, and hence all but finitely many of the $h\left(a_{i}\right)$ 's belong to $U$. Therefore $h$ is continuous at $p$ and $h$ is homeomorphism.

COROLLARY 1. If C is a crumpled cube then there exists an upper semi-continuous decomposition $G$ of $B(1)$ such that the intersection of each nondegenerate element of $G$ with the boundary of $B(1)$ in a one-point set, each nondegenerate element of $G$ is point-like, and $B(1) / G$ is homeomorphic to $C$.

Proof. This follows directly from Theorem 2 and Theorem 3.

Corollary 2. If $C$ and $D$ are crumpled cubes, $S$ is a tame 2-sphere in $S^{3}$, and $C$ and $D$ are sewn together along their boundaries, then there exists an upper semi-continuous decomposition $G$ of $S^{3}$ such that the intersection of each nondegenerate element of $G$ with $S$ is a one-point set and $S^{3} / G$ is homeomorphic to the sum of $C$ and $D$.

\section{Two examples.}

EXAMPLE 1. In [3] Bing constructed a 2-sphere $S$ with the property that $S$ is wild at each point and one of the complementary domains of $S^{3}-S$ is an open 3-cell. In this example, it is shown that if $C$ is the union of $S$ and the "bad" complementary domain of $S^{3}-S$ then the union of two copies of $C$ sewn together along their boundaries by the identity homeomorphism is topologically $S^{3}$.

The notation and terminology used will be the same as that used by Bing in [3]. Let $X$ denote the sum of two copies of $C$ sewn together along their boundaries by the identity homeomorphism. An upper semi-continuous decomposition $G$ of 
$S^{3}$ will be described. It will then be shown that $X$ is homeomorphic to $S^{3} / G$ and $S^{3} / G$ is homeomorphic to $S^{3}$.

$A$ decomposition of $S^{3}$. Let $S^{\prime}$ denote $\{(x, y, z): x=0\} \cup\{\infty\}$, and let $f$ be the homeomorphism defined by $f(x, y, z)=(-x, y, z)$ if $(x, y, z) \in E^{3}$ and $f(\infty)=\infty$. Let $f_{1}$ be a homeomorphism from $S^{3}$ onto $S^{3}$ such that $f_{1}\left[S_{0}\right]=S^{\prime}$ and for each $i, 1 \leqq i \leqq n$, let $A_{i}$ denote $f_{1}\left[T_{i}\right] \cup f f_{1}\left[T_{i}\right]$. Let $f_{2}$ be a homeomorphism from $S^{3}$ onto $S^{3}$ such that $f_{2} f_{1}\left[S_{1}\right]=S^{\prime}$ and if $x \in S^{3}-\bigcup_{i=1}^{n} A_{i}$ then $f_{2}(x)=x$, and for each $i$ and $\mathrm{j}, 1 \leqq i \leqq n, 1 \leqq j \leqq 15$, let $A_{i j}$ denote $f_{2}\left[T_{i j}\right] \cup f f_{2}\left[T_{i j}\right]$. The process is continued by induction. Let $A_{0}$ be $\left(\bigcup A_{i}\right) \cap\left(\bigcup A_{i j}\right) \cap\left(\bigcup A_{i j k}\right) \cap \cdots$. It is assumed that the $f_{i}^{\prime}$ 's are defined so that each component of $A_{0}$ is a tame arc that intersects $S^{\prime}$ in a one-point set.

Let $G$ denote $\left\{g: g\right.$ is a component of $A_{0}$ or there exists a $p$ in $S^{3}-A_{0}$ such that $\{p\}=g\}$. Then $G$ is an upper semi-continuous decomposition of $S^{3}$ and $S^{3} / G$ is homeomorphic to $X$.

To prove that $S^{3} / G$ is homeomorphic to $S^{3}$ it will be assumed that $S^{3}$ is $\{(x, y, z, w):|x|+|y|+|z|+|w|=1\}$ and $S^{\prime}$ is $\left\{(x, y, z, w):(x, y, z, w) \in S^{3}\right.$ and $w=0\}$.

If $B$ is the homeomorphic image of $A_{i}$ then $B_{i j}$ will denote the homeomorphic image of $A_{i j}, B_{i j k}$ denotes the homeomorphic image of $A_{i j k}$, etc.

Lemma 6. If $B$ is the homeomorphic image of $A_{i}$ in $S^{3}, F_{1}, \cdots, F_{m}$ are polyhedral disks such that (1) for each $i, 1 \leqq i \leqq m$, if $C$ is a component of $F_{i} \cap B$ then $C$ is a polyhedral disk, Int $C \subset$ Int $B$, and $B-C$ has exactly two components, the closure of each being a solid torus, and (2) for each pair of integers $i$ and $j, 1 \leqq i \leqq m, 1 \leqq j \leqq m$, and $i \neq j$, some component of $B-F_{i}$ contains $B \cap F_{j}$ and some component of $B-F_{j}$ contains $B \cap F_{i}$. Then there is a homeomorphism $h$ and a positive integer $j$ such that $h$ is from $S^{3}$ onto $S^{3}$, if $x \in S^{3}-B$ then $h(x)=x$, and for each $B_{i_{1} \ldots i_{j}}, h\left[B_{i_{1} \ldots i_{j}}\right]$ intersects at most one of the elements of $\left\{F_{1}, \cdots, F_{m}\right\}$.

Proof. Let $Q(m)$ be the statement that Lemma 6 is true if there are $m$ elements in $\left\{F_{1}, \cdots, F_{m}\right\}$.

If $h$ is the identity homeomorphism and $j=1$ then $Q(1)$ is true.

Assume $Q(k)$ is true for $k \leqq m-1$. Suppose $F_{1}, \cdots, F_{m}$ are polyhedral disks that satisfy the hypotheses of Lemma 6 . Let $\left\{C_{1}, \cdots, C_{k}\right\}$ be the set of components of $\left(\bigcup F_{i}\right) \cap B$ and assume the numbering is such that for each $j, 2 \leqq j \leqq k-1$, $\bigcup_{i<j} C_{i}$ and $\cup_{i>j} C_{i}$ are in different components of $B-C_{j}$. Then there exists integers $r, s, t$ and $v$ such that $\bigcup_{i=1}^{r} C_{i}=F_{s} \cap B$ and $\bigcup_{i=t}^{m} C_{i}=F_{v} \cap B$. Then there exist a homeomorphism $h^{\prime}$ such that (1) $\mathrm{h}^{\prime}$ is from $S^{3}$ onto $S^{3}$, (2) if $x \in S^{3}-B$ then $h^{\prime}(x)=x$, (3) for each $i$ and $j, 1 \leqq j \leqq 15$, and $1 \leqq i \leqq m$, if $C$ is a component of $h^{\prime}\left[B_{j}\right] \cap F_{i}$, then $C$ is a polyhedral disk, Int $C \subset \operatorname{Int} B$, and $h^{\prime}\left[B_{j}\right]-C$ has exactly two components, the closure of each being a solid torus, (4) for each triple 
of integers $i, j, k, 1 \leqq i \leqq m, 1 \leqq j \leqq m, 1 \leqq k \leqq 15$, and $i \neq j$, such that $F_{i}$ and $F_{j}$ intersect $h^{\prime}\left[B_{k}\right]$, some component of $h^{\prime}\left[B_{k}\right]-F_{j}$ contains $F_{i} \cap h^{\prime}\left[B_{k}\right]$ and some component of $h^{\prime}\left[B_{k}\right]-F_{i}$ contains $F_{j} \cap h^{\prime}\left[B_{k}\right]$, and (5) for each $k$, $1 \leqq k \leqq 15, h^{\prime}\left[B_{k}\right]$ intersects at most $m-1$ of the elements of $\left\{F_{1}, \cdots, F_{m}\right\}$. Hence for each $k, 1 \leqq k \leqq 15$, there exists an integer $z \leqq m-1$ such that $h^{\prime}\left[B_{k}\right]$ satisfies the hypotheses of Lemma 6 and $h^{\prime}\left[B_{k}\right]$ intersects at most $z$ of the elements in $\left\{F_{1}, \cdots, F_{m}\right\}$. Therefore there exists a homeomorphism $h_{k}$ and an integer $j(k)$ such that $h_{k}$ is from $S^{3}$ onto $S^{3}$, if $x \in S^{3}-h^{\prime}\left[B_{k}\right]$ then $h_{k}(x)=x$, and for each $h^{\prime}\left[B_{i_{1} \ldots i_{j(k)}}\right], h_{k}\left[h^{\prime}\left[B_{i_{1} \ldots i_{j(k)}}\right]\right]$ intersects at most one of the elements in $\left\{F_{1}, \cdots, F_{m}\right\}$. Let $h=h_{1} h_{2} \cdots h_{15} h^{\prime}$ and let $j=\max \{j(k): 1 \leqq k \leqq 15\}$. Then $h$ and $j$ satisfy the conclusion of Lemma 6. Hence $Q(m)$ is true, and the Lemma is proved.

Lemma 7. If $B$ is the homeomorphic image of $A_{i}$ in $S^{3}$ and $P_{1}, \cdots$, and $P_{m}$ are disjoint polyhedral 2-spheres in $S^{3}$, then there is a homeomorphism $h$ and a positive integer $q$ such that $h$ is from $S^{3}$ onto $S^{3}$, if $x \in S^{3}-B$ then $h(x)=x$, and for each $B_{i_{1} \ldots i_{q}}, h\left[B_{i_{1} \ldots i_{q}}\right]$ intersects at most one of the elements in $\left\{P_{1}, \cdots, P_{m}\right\}$.

Proof. There exists a homeomorphism $k$ from $S^{3}$ onto $S^{3}$ such that if $x \in S^{3}-B$ then $k(x)=x$, for each $i$ and $j, 1 \leqq j \leqq 15$, and $1 \leqq i \leqq n, k\left[B_{i j}\right]$ is polyhedral and $k\left[B_{i j}\right], P_{1}, \cdots$, and $P_{m}$ are in relative general position, and for each $i$ and $j$, $1 \leqq j \leqq 15$, and $1 \leqq i \leqq n$, if $C$ is a component of $k\left[B_{i j}\right] \cap\left(\bigcup_{i=1}^{m} P_{i}\right)$, then $C$ is a polyhedral disk, Int $C \subset \operatorname{Int} k\left[B_{i j}\right]$, and $k\left[B_{i j}\right]-C$ has exactly two components, the closure of each being a solid torus. Let $\left\{F_{1}, \cdots, F_{r}\right\}$ be the collection of components of $\left(\bigcup_{j=1}^{15} k\left[B_{i j}\right]\right) \cap\left(\bigcup_{i=1}^{m} P_{i}\right)$.

Lemma 7 then follows from Lemma 6.

Suppose the 2-spheres $P_{1}, \cdots$, and $P_{m}$ in the hypothesis of Lemma 7 satisfy the following conditions: (1) For each $r, 1 \leqq r \leqq m$, there exists a real number $t(r)$ such that $P_{r}=\left\{(x, y, z, w):(x, y, z, w) \in S^{3}\right.$ and $\left.w=t(r)\right\}$ and (2) For each $r$, $2 \leqq r \leqq m, t(r)>t(r-1)$. If $v$ is a real number such that for each $r, 2 \leqq r \leqq m$, $t(r)-t(r-1)<v$, then the homeomorphism $h$ in Lemma 7 can be constructed such that if $M$ is a subset of $h\left[B_{i_{1} \ldots i_{q}}\right]$, then the diameter of $M$ is less than $3 v$. Therefore Lemma 7 can be strengthened to obtain the following result.

Lemma 8. If $B$ is the homeomorphic image of $A_{i}$ in $S^{3}$ and $e$ is a positive number, then there exists a homeomorphism $h$ and a positive integer $q$ such that $h$ is from $S^{3}$ onto $S^{3}$, if $x \in S^{3}-B$ then $h(x)=x$, and for each $B_{i_{1} \ldots i q}$, the diameter of $h\left[B_{i_{1} \ldots i q}\right]$ is less than $e$.

THEOREM 4. If $G$ is the upper semi-continuous decomposition of $S^{3}$ whose nondegenerate elements are the components of $A_{0}$, then $S^{3} / G$ is homeomorphic to $S^{3}$.

Proof. It follows from Lemma 8 that for each positive integer $j$ there exists a homeomorphism $t_{j}$ and an integer $q(j)$ such that $t_{j}$ leaves $S^{3}-\bigcup A_{i_{1} \ldots i_{j}}$ pointwise 
fixed, and for each nondegenerate element $g$ of $G$ the diameter of $t_{j}[g]$ is at most $1 / j$. It follows that $S^{3}$ is homeomorphic to $S^{3} / G$.

Example 2. A "nice" crumpled cube $D$ with the property that the union of two copies of $D$ sewn together along their boundaries by the identity homeomorphism is not homeomorphic to $S^{3}$.

Description. Let $P$ be the 2-sphere $\left\{(x, y, z) \in E^{3}: x=0\right\} \cup\{\infty\}$ and let $K$ be the 3-cell $\left\{(x, y, z) \in E^{3}: x \geqq 0\right\} \cup\{\infty\}$. Let $J$ be the Fox-Artin arc of Figure 6, contained in Int $K$ except for its left endpoint which lies on $P$. Let $G^{\prime}$

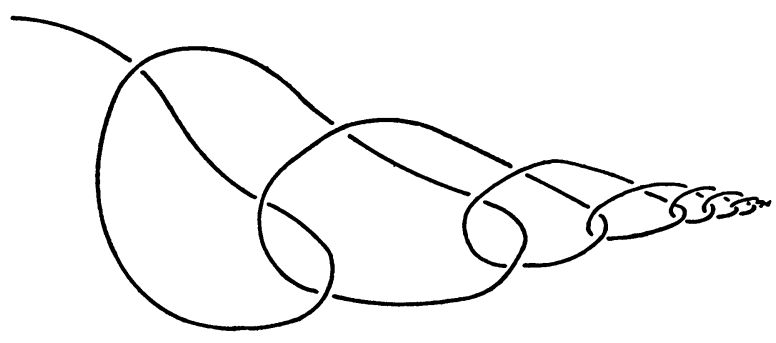

FIGURE 6

be the upper semi-continuous decomposition of $S^{3}$ whose only nondegenerate element is $J$. Since $J$ is cellular, $S^{3} / G^{\prime}$ is homeomorphic to $S^{3}$ and the projection map $\phi$ from $S^{3}$ onto $S^{3} / G^{\prime}$ is a homeomorphism on $P$. Hence $\phi[K]$ is a crumpled cube.

Let $G$ be the upper semi-continuous decomposition of $S^{3}$ whose only nondegenerate element is $\bigcup\{(x, y, z):(|x|, y, z) \in J\}$. Then $S^{3} / G$ is homeomorphic to the union of two copies of $\phi[K]$ sewn together along their boundaries by the identity homeomorphism. It follows from Example 1.3 of [8] that $S^{3} / G$ is not homeomorphic to $S^{3}$.

\section{BIBLIOGRAPHY}

1. B. J. Ball, The sum of two solid horned spheres, Ann. of Math. (2) 69 (1959), 253-257.

2. R. H. Bing, A homeomorphism between the 3-sphere and the sum of two solid horned spheres, Ann. of Math. 56 (1952), 354-362.

3. —- A wild surface each of whose arcs is tame, Duke, Math. J. 28 (1961), 1-15.

4. —-, Approximating surfaces from the side, Ann. of Math. (2) 77 (1963), 145-192.

5. - Conditions under which a surface in $E^{3}$ is tame, Fund. Math. 47 (1959), 105-139.

6. —_, Mimeographed Notes, Topology Institute, University of Georgia, Summer, 1961.

7. B. G. Casler, On the sum of two solid Alexunder horned spheres, Notices Amer. Math. Soc. 9 (1962), 108.

8. R. H. Fox and E. Artin, Some wild cells and spheres in three dimensional space, Ann. of Math. (2) 49 (1948), 979-990.

9. Norman Hosay, The sum of a real cube and a crumpled cube is $S^{3}$, Notices Amer. Math. Soc. 10 (1963), 666.

UNIVERSITY OF IOWA,

IowA City, IowA 